\title{
Sustained maternal smoking-associated changes in the physico-chemical properties of fetal RBC membranes might serve as early markers for vascular comorbidities
}

\author{
Gábor Balogh $^{\mathrm{a}, 1}$, Payal Chakraborty ${ }^{\mathrm{b}, 1}$, Krisztina N. Dugmonits ${ }^{\mathrm{b}, 1}$, Mária Péter ${ }^{\mathrm{a}, 1}$, Attila G. Végh , \\ László Vígh ${ }^{\mathrm{a}}$, Edit Hermesz ${ }^{\mathrm{b}, *}$ \\ ${ }^{a}$ Institute of Biochemistry, Biological Research Centre, Hungarian Academy of Sciences, Szeged, Hungary \\ ${ }^{\mathrm{b}}$ Department of Biochemistry and Molecular Biology, Faculty of Science and Informatics, University of Szeged, Szeged, Hungary \\ ${ }^{\mathrm{c}}$ Institute of Biophysics, Biological Research Centre, Hungarian Academy of Sciences, Szeged, Hungary
}

\section{A R T I C L E I N F O}

\section{Keywords:}

Deformability

Fetal RBC

Lipid

Membrane damage

Peroxynitrite

Smoking

\begin{abstract}
A B S T R A C T
Maternal smoking-induced congenital heart and microvascular defects are closely associated with the impaired functioning of the in-utero feto-placental circulation system. Current groundbreaking facts revealed intimate crosstalk between circulating red blood cells (RBCs) and the vascular endothelium. Thus, RBCs have become the protagonists under varied pathological and adverse pro-oxidative cellular stress conditions. We isolated and screened fetal RBCs from the arterial cord blood of neonates, born to non-smoking (RBC-NS) and smoking mothers (RBC-S), assuming that parameters of fetal RBCs are blueprints of conditions experienced in-utero. Using atomic force microscopy and mass spectrometry-based shotgun lipidomics in the RBC-S population we revealed induced membrane stiffness, loss in intrinsic plastic activities and several abnormalities in their membrane-lipid composition, that could consequently result in perturbed hemodynamic flow movements. Altogether, these features are indicative of the outcome of neonatal microvascular complications and suggest unavailability for the potential rescue mechanism in cases of vascular endothelium impairment due to altered membrane integrity and rheological properties.
\end{abstract}

\section{Introduction}

More than 4800 compounds are present in the particulate and vapor phases of cigarette smoke [1], and many of these compounds constitute human health risk factors [2]. The adverse effects are mainly derived from toxic materials due to enhanced oxidative damage in critical biological substances [3]. Around $20-30 \%$ of women continue to smoke during full-term pregnancy [4]. Based on epidemiological and toxicological studies, smoking can exert a marked impact on the outcome of in-utero development [5]. Since 1959, observational clinical studies have shown an escalation towards non-chromosomal childbirth anomalies. The upsurge of congenital heart defects lies with vascular endothelial damage and complexities like ventricular and atrial septal defects, right and left ventricular outflow tract obstruction and conotruncal defects, which are closely associated with a higher rate of maternal smoking during pregnancy [6]. Therefore, in the current clinical setup neonatal heart defects with microvascular disorders caused by intensive maternal smoking became growing health concern issues.

During pregnancy the placenta and the umbilical cord form vital temporary organs to sustain in-utero conditions. The intact functioning of the feto-placental unit gets adapted to the state of increased metabolic and oxygen demands with a supply of oxygen, nutrient substrates and effective elimination of the metabolic waste [7]. The umbilical cord

Abbreviations: AFM, atomic force microscopy; CAT, catalase; Chol, cholesterol; DBI, double bond index; FACS, fluorescence activated cell sorting; $\mathrm{H}_{2} \mathrm{O}_{2}$, hydrogen peroxide; 4-HNE, 4-hydroxynonenal; NO, nitric oxide; NOS3, nitric oxide synthase; LPC, lysophosphatidylcholine; ONOO ${ }^{-}$, peroxynitrite; PC, phosphatidylcholine; PE, phosphatidylethanolamine; PI, phosphatidylinositol; PS, phosphatidylserine; PL, phospholipids; PUFA, polyunsaturated fatty acid; RBC, red blood cells; RBC-S, RBCs of the developing fetus born to smoking mother; RBC-NS, RBCs of the developing fetus born to non-smoking mother; ROS, reactive oxygen species; SM, sphingomyelin; SOD, superoxide dismutase

* Corresponding author at: Department of Biochemistry and Molecular Biology, Faculty of Science and Informatics, University of Szeged, H-6726 Szeged, Középfasor 52, Hungary.

E-mail address: hermesz@bio.u-szeged.hu (E. Hermesz).

${ }^{1}$ Contributed equally to this work. 
lacks innervation so the vascular tone and integrity majorly depend on the cord endothelial-derived nitric oxide (NO) via the functional nitric oxide synthase (NOS3). In cases of active maternal smoking the fetal oxygenation deteriorates by the infiltration of extrinsic harmful toxic elements through the placental barrier into the feto-placental circulation. The feto-placental unit with low antioxidant and immunologic competence is highly sensitive to long-term exposition towards harmful materials, which cause an inevitable loss of redox balance with development of oxidative stress condition. It induces proinflammatory stimulus, causing vascular endothelium dysfunctioning with placental insufficiency, restricted hemodynamic flow, deprivation in the oxygen and nutrient levels [8,9].

Until recently, pathological endothelial dysfunctions were correlated with vessel wall constriction, inflammation, thrombosis and proliferation. The added groundbreaking facts on the human circulatory red blood cells (RBCs) with their 'erythrocrine function' under normal physiological condition and 'erythropathy' in disease states exclusively define a new functional role of RBCs [10-12]. Besides carrying oxygen, transmission of metabolic gases and nutrients to the tissues, RBCs can synthesize and export NO by an active RBC-NOS isoform and liberate ATP with a well-equipped endogenous redox buffering system. There are clear evidences in adults that, under disease states, RBCs show excess levels of reactive oxygen species (ROS), altered protein profile and due to an intimate crosstalk with the vascular endothelium, they synergize the process of vascular impairment [10-12].

In the context of hemorheological and hemodynamic flow properties, several studies were conducted on RBCs from both healthy and patient volunteers with wide range of inherited red cell disorders to explore the underlying molecular processes in the red cell membrane. The uniqueness of human RBC is majorly characterized by its plasma membrane that promotes all of its diverse antigenic, transport and mechanical characteristics. The unique RBC membrane envelops a composite structure of phospholipids (PLs) clustered together with an elastic network of skeletal proteins via transmembrane proteins. The lipid bilayer eventually regulates the membrane fluidity/deformability of RBCs, an intrinsic nanomechanical property, which allows them to traverse across various shear stress and continuously changing flow conditions along the vasculature [13,14]. Excess formation of cytotoxic oxidants and NO are important indicators of various pathological processes, which lead to redox homeostasis imbalance and therefore might deleteriously affect the nanomechanical properties of RBCs [15].

In adults, cardiovascular complications have been attributed to lifestyle or genetic disorders but there is no clear understanding to identify the key risks factors associated with rising neonatal microvascular comorbidities. Though advanced non-invasive biomedical devices like Doppler and ultrasound can easily detect any kind of abnormalities in the umbilical circulation under in-utero conditions, the underlying biochemical and molecular insights on how tobacco smoke affects fetal development are missing. The basic purpose of our study was to elucidate that how the nanomechanical activities subsequently influence the feto-placental hemodynamic flow conditions under severe oxidative stress conditions; these can represent key factors in the prognosis of neonatal microvascular complications. We assumed that the parameters of fetal RBCs derived from arterial cord blood are blueprints of conditions experienced in-utero. Thus, we aimed to directly investigate and map the continuous effect of tobacco exposure on the biochemical and biophysical characteristics of fetal RBCs isolated from the arterial cord blood at the time of birth.

\section{Material and methods}

\subsection{Human samples}

In accordance to the principles outlined in the Declaration of Helsinki and signed consent of the pregnant mothers, fetal blood samples were collected at the Department of Obstetrics and Gynaecology,
University of Szeged, Hungary. The Ethics Committee of the Department approved the study protocol (16/2014; Investigation of oxidative stress markers in maternal and neonatal blood samples). RBCs were isolated from the arterial cord blood of neonates, born to nonsmoking (RBC-NS, $\mathrm{n}=60$ ) and heavy smoker (RBC-S, $\mathrm{n}=55$ ) mothers (at least 10 cigarettes per day). Age below 18 years, gestational age $<37$ weeks, gestational diabetes, infection or inflammatory conditions or disorders such as cardiovascular diseases, complications during delivery, malformations or evidence of genetic disorders were the exclusion factors. The nutritional status of the mothers during pregnancy was satisfactory; no case of malnutrition occurred.

Blood samples were centrifuged at $200 \mathrm{~g}$ for $5 \mathrm{~min}$ at $20^{\circ} \mathrm{C}$ and the lowest two-third portion from the RBC phase was collected. Consecutive washing of the RBCs was done with 2 volumes of isotonic saline solution at pH-7.0. The purity of the RBC preparation was > $95 \%$. The homogeneity of the RBC preparation was confirmed by immunostaining with RBC-specific mouse anti-Glycophorin A antibody. Samples for immunohistochemistry, spectrophotometric and atomic force microscopy studies were processed immediately. For lipidomic analysis, RBC ghost membrane preparation was carried out immediately and stored at $-80{ }^{\circ} \mathrm{C}$ until further processing.

\subsection{Determination of hydrogen peroxide $\left(\mathrm{H}_{2} \mathrm{O}_{2}\right)$ and peroxynitrite $\left(\mathrm{ONOO}^{-}\right)$level}

The RBCs were haemolysed by the addition of distilled water at a ratio of 1:9. The hemolysate of the samples were diluted in $50 \mathrm{mM} \mathrm{PB}$ (pH-6.0) at a ratio of 1:250. The assay was performed according to Villegas and Gilliland [16]. Serial dilutions of $\mathrm{H}_{2} \mathrm{O}_{2}$ between 27.5 and $440 \mu \mathrm{M}$ were prepared and used as a standard. Reaction mixture without blood sample was used as a reference. Samples were incubated with $0.1 \%(\mathrm{w} / \mathrm{v})$ horseradish peroxidase in $50 \mathrm{mM} \mathrm{PB}(\mathrm{pH}-6.0)$ and $1 \%$ (w/v) o-dianisidine in methanol for colour development, at $37{ }^{\circ} \mathrm{C}$ for $10 \mathrm{~min}$. The reaction was stopped by $4 \mathrm{~N} \mathrm{HCl}$. The $\mathrm{H}_{2} \mathrm{O}_{2}$ concentration was determined spectrophotometrically at $400 \mathrm{~nm}$, using GENESYS $10 \mathrm{~S}$ UV-Vis spectrophotometer (Thermo Fischer Scientific, Madison, WI, USA) and the results were calculated according to the standard curve and normalized with protein concentration ( $\mu \mathrm{mol} / \mathrm{mg}$ protein).

Measurement of $\mathrm{ONOO}^{-}$level was performed at $302 \mathrm{~nm}$. Each hemolysate was diluted with $1 \mathrm{M} \mathrm{NaOH}$ solution in a ratio of 1:250. The increase of absorbance was measured until it reached a stable equilibrium, then the sample was added into $100 \mathrm{mM} \mathrm{PB} \mathrm{(pH} \mathrm{7.4)} \mathrm{in} \mathrm{the} \mathrm{same}$ ratio as the reference. At this neutral $\mathrm{pH}$, the $\mathrm{ONOO}^{-}$decomposes, and the decrease in absorbance can be followed till equilibrium [17]. According to the Lambert-Beer law the $\mathrm{ONOO}^{-}\left(\varepsilon_{\mathrm{ONOO}^{-}}\right.$ $=1670 \mathrm{M}^{-1} \mathrm{~cm}^{-1}$ ) concentration was calculated by the absorbance difference at two distinct $\mathrm{pH}$ values and normalized for protein concentration, where the total protein levels were measured following the protocol of Lowry et al. [18].

\subsection{Enzyme activity measurements}

Superoxide dismutase, SOD (EC 1.15.1.1) activity was determined on the basis of the inhibition of the epinephrine-adrenochrome autoxidation [19]. The hemolysate of the sample was used directly, without further dilution. Spectrophotometric measurement was carried out at $480 \mathrm{~nm}$ using GENESYS 10S UV-Vis spectrophotometer (Thermo Fisher Scientific, Madison, WI, USA). Results were expressed in U/mg protein.

Catalase, CAT (EC 1.11.1.6) activity was determined by spectrophotometrical study at $240 \mathrm{~nm}$ [20] and CAT specific activity was expressed in Bergmeyer units (BU)/g protein (1 BU = decomposition of $1 \mathrm{~g} \mathrm{H}_{2} \mathrm{O}_{2} / \mathrm{min}$ at $25{ }^{\circ} \mathrm{C}$ ).

\subsection{Lipidomic analysis}

Lipid standards were obtained from Avanti Polar Lipids (Alabaster, 
AL, USA). The solvents used for extraction and for mass spectrometric (MS) analyses were of liquid chromatographic grade from Merck (Darmstadt, Germany) and Optima LCMS grade from Thermo Fisher Scientific (Waltham, MA, USA). All other chemicals were purchased from Sigma-Aldrich (Steinheim, Germany) and were of the best available grade.

Resuspended RBC ghost pellet was subjected to one-phase methanolic extraction [21]. $20 \mu \mathrm{L} \mathrm{RBC}$ ghost suspension was sonicated in $1 \mathrm{~mL}$ methanol (containing $0.001 \%$ butylated hydroxytoluene as antioxidant) for $5 \mathrm{~min}$, shaken for $5 \mathrm{~min}$, and centrifuged at 10,000 $\times \mathrm{g}$ for $5 \mathrm{~min}$. The supernatant was transferred into a new Eppendorf tube and stored at $-20{ }^{\circ} \mathrm{C}$ until MS analysis.

MS-based lipidomic analyses were performed on an Orbitrap Fusion Lumos instrument (Thermo Fisher Scientific, Bremen, Germany) equipped with a TriVersa NanoMate robotic nanoflow ion source (Advion BioSciences, Ithaca, NY) using chips with spraying nozzles having a diameter of $5.5 \mu \mathrm{m}$, as described in [22]. The ion source was controlled by Chipsoft 8.3.1 software. The ionization voltages were $+1.3 \mathrm{kV}$ and $-1.9 \mathrm{kV}$ in positive and negative mode, respectively, and the backpressure was set at $1 \mathrm{psi}$ in both modes. The temperature of the ion transfer capillary was $330{ }^{\circ} \mathrm{C}$. Acquisitions were performed at the mass resolution $R_{m / z} 200=240,000$.

Phosphatidylcholine (PC, diacyl and PC-O, alkyl-acyl), lysophosphatidylcholine (LPC), and sphingomyelin (SM) were detected and quantified using the positive ion mode. Cholesterol (Chol) was derivatized according to Liebisch et al. [23] and analysed in the positive ion mode. Phosphatidylethanolamine (PE, diacyl and PE-Pl, alkenyl-acyl), phosphatidylinositol (PI), phosphatidylserine (PS), the lyso derivatives LPE, LPI, LPS, phosphatidic acid (PA) and ceramide (Cer) were detected and quantified using the negative ion mode. For MS analysis, $10 \mu \mathrm{L}$ lipid extract was diluted with $140 \mu \mathrm{L}$ infusion solvent mixture (chloroform: methanol: isopropanol 1:2:1, by vol.) containing an internal standard mix (66 pmol PC d31-16:0/18:1, 23 pmol PE d31-16:0/ 18:1, 10 pmol PI d31-16:0/18:1, 18 pmol PS d31-16:0/18:1, 1 pmol PA d31-16:0/18:1, 24 pmol SM d18:1/17:0,2 pmol Cer d18:1/17:0). Next, the mixture was halved, and $5 \%$ dimethylformamide (additive for the negative ion mode) or $3 \mathrm{mM}$ ammonium chloride (additive for the positive ion mode) were added to the split sample halves. Lipid species were identified by LipidXplorer software [24]. The mass tolerance was $3 \mathrm{ppm}$. Data files generated by LipidXplorer queries were further processed by in-house Excel macros.

The double bond index (DBI) was calculated as $\Sigma(\mathrm{mol} \%$ of unsaturated fatty acids in a given lipid class $\times$ number of double bonds of each unsaturated fatty acid) / 100 .

Lipid species are given as sum formulas according to Liebisch et al. [25]. For glycerolipids, e.g., PC (34:1), the total number of carbons followed by double bonds for all chains are indicated. For sphingolipids, e.g., SM (34:1:2), first the total number of carbons in the long chain base and the fatty acid (FA) moiety then the sum of double bonds in the long chain base and the FA moiety are indicated followed by the sum of hydroxyl groups in the long chain base and the FA moiety. Lipidomic results are expressed as mol\% of membrane lipids that consisted of glycerophospholipids, sphingolipids and Chol.

\subsection{Fluorescence-activated cell sorting (FACS) analysis}

Fixation and staining of RBCs were performed using standard protocols [26]. Cells were fixed with $4 \%(\mathrm{w} / \mathrm{v})$ paraformaldehyde in $0.05 \mathrm{M}$ phosphate buffer (PB) at pH-7.2 for FACS analysis at $4{ }^{\circ} \mathrm{C}$ for $60 \mathrm{~min}$. After consecutive washing with $\mathrm{PB}$, they were subjected to permeabilization by $0.1 \%$ Triton-X100 and further incubated for $1 \mathrm{~h}$ in PB containing $1 \%$ bovine serum albumin and $10 \%$ normal goat serum to block non-specific antibody binding. RBCs were immunolabelled with mouse anti-Glycophorin A (CD235a) (1:50 dilution, MA5-12484, Thermo Fisher Scientific, Madison, WI, USA), rabbit anti-NOS3 antibody (1:100 dilution, ab5589, Santa Cruz Biotechnology Inc., Dallas,
TX, USA) and mouse anti 4-hydroxy-2-nonenal antibody (4-HNE) (1:100 dilution, ab48506, Abcam, Cambridge, UK), by single or double staining, at $4{ }^{\circ} \mathrm{C}$ for overnight. Incubations with the primary antibodies were followed by washing and incubation with goat anti-mouse Alexa ${ }^{\circledR} 647$ and/or with goat anti-rabbit Alexa ${ }^{\circledR} 488$-conjugated secondary antibodies for $1 \mathrm{~h}$ at room temperature. After washing, RBCs were processed for quantitative analysis (FACS, BD FACS Calibur ${ }^{\mathrm{TM}}$, BD Biosciences). The FACS data were analysed using FlowJo_V10 software tool.

\subsection{Atomic force microscopy}

\subsubsection{Instrumentation}

All elasticity experiments were carried out with Asylum Research MFP-3D atomic force microscope (Asylum Research, Santa Barbara, CA; driving software IgorPro 6.32A, Wavemetrics), being mounted on a Zeiss Axiovert 200 optical microscope for initial positioning. The experiments were performed with a V-shaped tip mounted on a gold coated silicon nitride rectangular cantilever (BL RC150VB-A), nominal spring constant of $30 \mathrm{pN} / \mathrm{nm}$ and resonant frequency of $37 \mathrm{kHz}$. Prior to experiments, spring constant of each cantilever was calibrated [27,28].

$20 \mu \mathrm{L}$ of freshly prepared RBC solution was dropped onto $1 \mathrm{mg} / \mathrm{mL}$ polyethyleneimine coated Petri Lid surface. Measurements were carried out in $0.9 \% \mathrm{NaCl}$ solution. With the help of the optical microscope, RBCs were positioned for elastic mapping. In each case, $15 \times 15 \mu \mathrm{m}^{2}$ area was selected, divided into 40 lines by 40 points and at every point a force distance curve was recorded at $8 \mu \mathrm{m} / \mathrm{s}$ speed, having maximal load of $0.5 \mathrm{nN}$ and $2 \mathrm{kHz}$ sampling frequency. Nanomechanical properties were extracted using a custom written MatLab ${ }^{\circledR}$ routine, described elsewhere [29].

\subsubsection{Data analysis}

Elastic and plastic properties of the studied RBCs were compared based on the calculated elastic and remanent work for each force curve. All parameters were extracted with the help of a custom-made MatLab ${ }^{\circledR}$ (MathWorks) routine. The elastic index was defined as the ratio between the elastic and remanent work [29,30]. Pseudo coloured three dimensional maps were reconstructed using the driving software of the instrument. Data is presented as average \pm standard deviation [31].

\subsection{Statistical analysis}

For $\mathrm{ONOO}^{-}, \mathrm{H}_{2} \mathrm{O}_{2}$, SOD, CAT and FACS measurements, statistical analyses were calculated with one-way analysis of variance (ANOVA) (GraphPad Statistical Software version 4.0) using Newman-Keuls multiple comparison test. Significant differences were accepted at ${ }^{*} \mathrm{p}<0.05,{ }^{* *} \mathrm{p}<0.01,{ }^{* * *} \mathrm{p}<0.001$ and ${ }^{* * * *} \mathrm{p}<0.0001$. Atomic force microscopy data were analysed by Kruskal-Wallis rank test, since this test does not presume normal distribution of values and gives accurate results for relatively small sample sizes as well. Significant differences were accepted at ${ }^{*} \mathrm{p} \leq 0.05$. For lipidomic data, significance was determined by Student's $t$-test and was accepted at $\mathrm{p}<0.05$. Orthogonal partial least squares discriminant analysis (OPLS-DA) was performed and validated using MetaboAnalyst [32].

\section{Results}

\subsection{Increased $\mathrm{ONOO}^{-}$level in the RBC-S population indicates ROS overproduction}

Sustained smoking tends to enhance the intrinsic pro-oxidant content. In RBCs originated from neonates born to smoking mother a significant $\sim 1.5-2$-fold increase was detected in the level of $\mathrm{ONOO}^{-}$ (Fig. 1A), while the level of $\mathrm{H}_{2} \mathrm{O}_{2}$ and the activities of the enzymes responsible for its synthesis (superoxide dismutase, SOD) and degradation (catalase, CAT) showed decreasing tendencies, but these were 


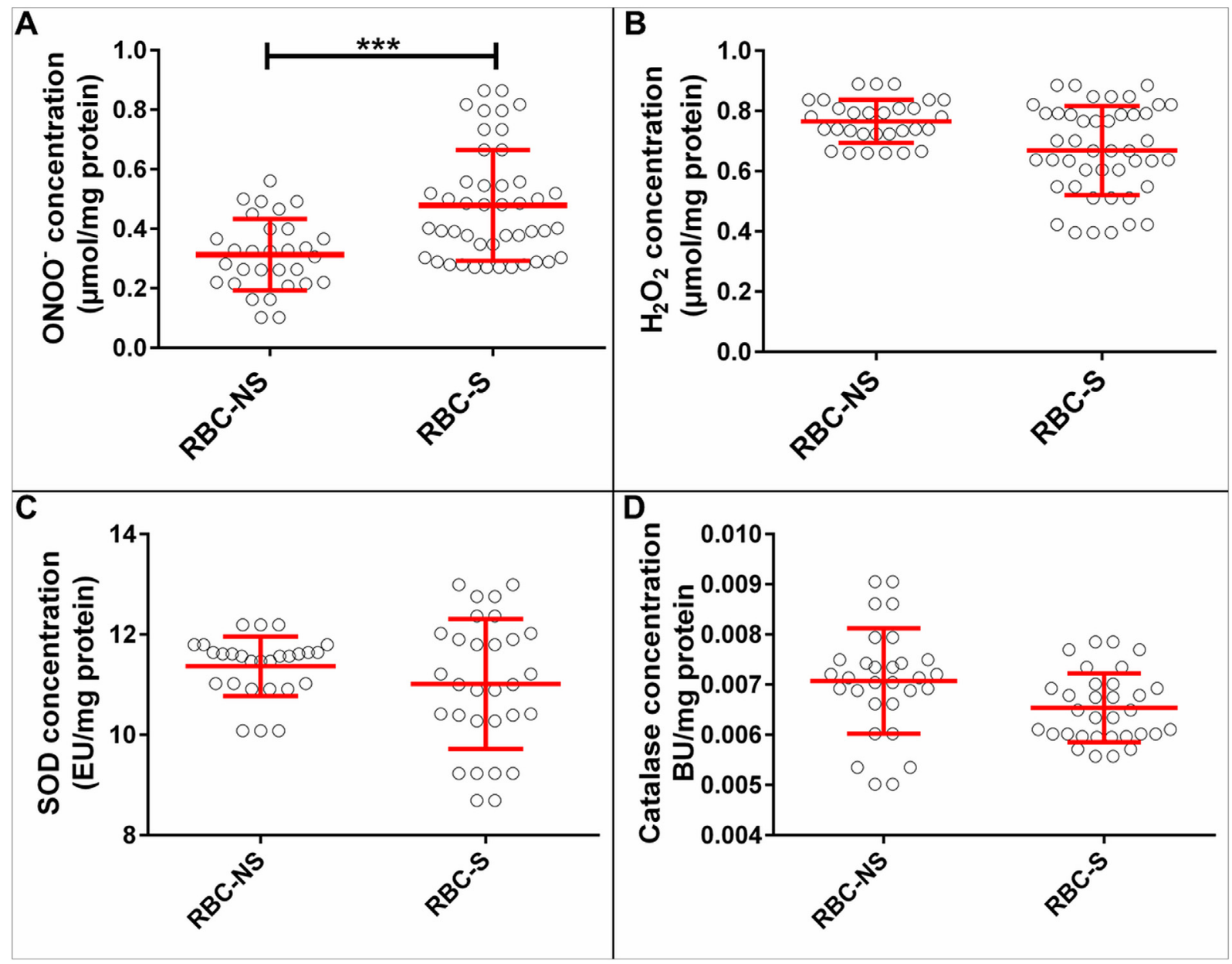

Fig. 1. Spectrophotometric measurement on strong oxidants and antioxidant enzymes in RBC-NS and RBC-S populations.

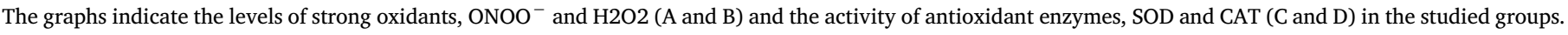

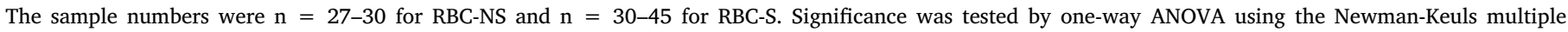
comparison test and accepted at $* * * \mathrm{p}<0.001$.

not significant (Fig. 1B, C and D).

\subsection{Nanomechanical parameters indicate altered rheological state of $R B C$ - $S$ group}

High resolution direct measurement of RBC deformability is a challenging task. To distinguish the nanomechanical characteristics of the RBC-NS and RBC-S populations, we used atomic force microscopybased force spectroscopy [33] and calculated the indenting energy (Elastic Work) and remanent energy (Plastic Work) using a custommade MatLab ${ }^{\circledR}$ routine on AFM data. As all force curves were recorded using the same maximal load, the indenting energy is a suitable parameter for comparing the elastic properties of the studied cells. The cells of RBC-S origin required slightly lower elastic work to be indented at the same extent than the RBC-NS cells (Fig. 2A, B and E $\mathrm{x}$ axis), while considerably lower values $(\sim 1 / 2)$ were recorded for remanent, i.e., plastic work after indentation (Fig. 2C, D and E y axis). As a consequence, the ratio of remanent and indenting energies, named Elastic index, was significantly lower for the RBC-S population (Fig. 2F).

\subsection{Lipidomic analysis reveals alterations in the RBC-S plasma membrane organization}

To assess the alterations that may underlie the large deformability difference between the RBC-NS and RBC-S populations, we hypothesized the contribution of an altered lipid composition of the RBC plasma membrane. To test this hypothesis, we applied comprehensive electrospray ionization mass spectrometric measurements on RBC membrane lipid extracts. Our high sensitivity, high resolution shotgun lipidomic approach allowed the identification and quantification of $c a .150$ lipid molecular species encompassing 14 lipid classes (Supplementary Lipid Table). To obtain an overview of the lipidome changes, the MS data, expressed as mol\% of membrane lipids, were subjected to multivariate statistical analysis. In orthogonal partial least squares discriminant analysis (OPLS-DA) the RBC-NS and RBC-S groups were well separated (Fig. 3), and permutation tests $(n=1000)$ returned good predictability ( $22=0.58, \mathrm{p}<0.001$ ) as well as high goodness-of-fit value $(\mathrm{R} 2=0.94, \mathrm{p}<0.001)$ for the model.

In order to identify appropriate differences of relevance, data were grouped for changes in lipid classes, leaflet asymmetry, degree of unsaturation, and susceptibility to oxidation. Based on such groupings, the following trends were revealed. The Chol/PL ratio increased in the membranes of the RBC-S group as compared to the RBC-NS (Fig. 4A). The ratio of the outer leaflet-dominant choline-containing PC + SM versus the inner leaflet-dominant PE + PS + PI was found to be different between the RBC-NS and RBC-S groups (Fig. 4B); of note, the major contribution could be attributed to the decrease of PS level (Fig. 4C). The average degree of unsaturation of the RBC membrane lipids was also calculated and characterized by the double bond index (DBI). DBI was found to be significantly decreased in the RBC-S population as compared to the RBC-NS (Fig. 4D). And finally, we detected pronounced reductions in the levels of several polyunsaturated fatty 


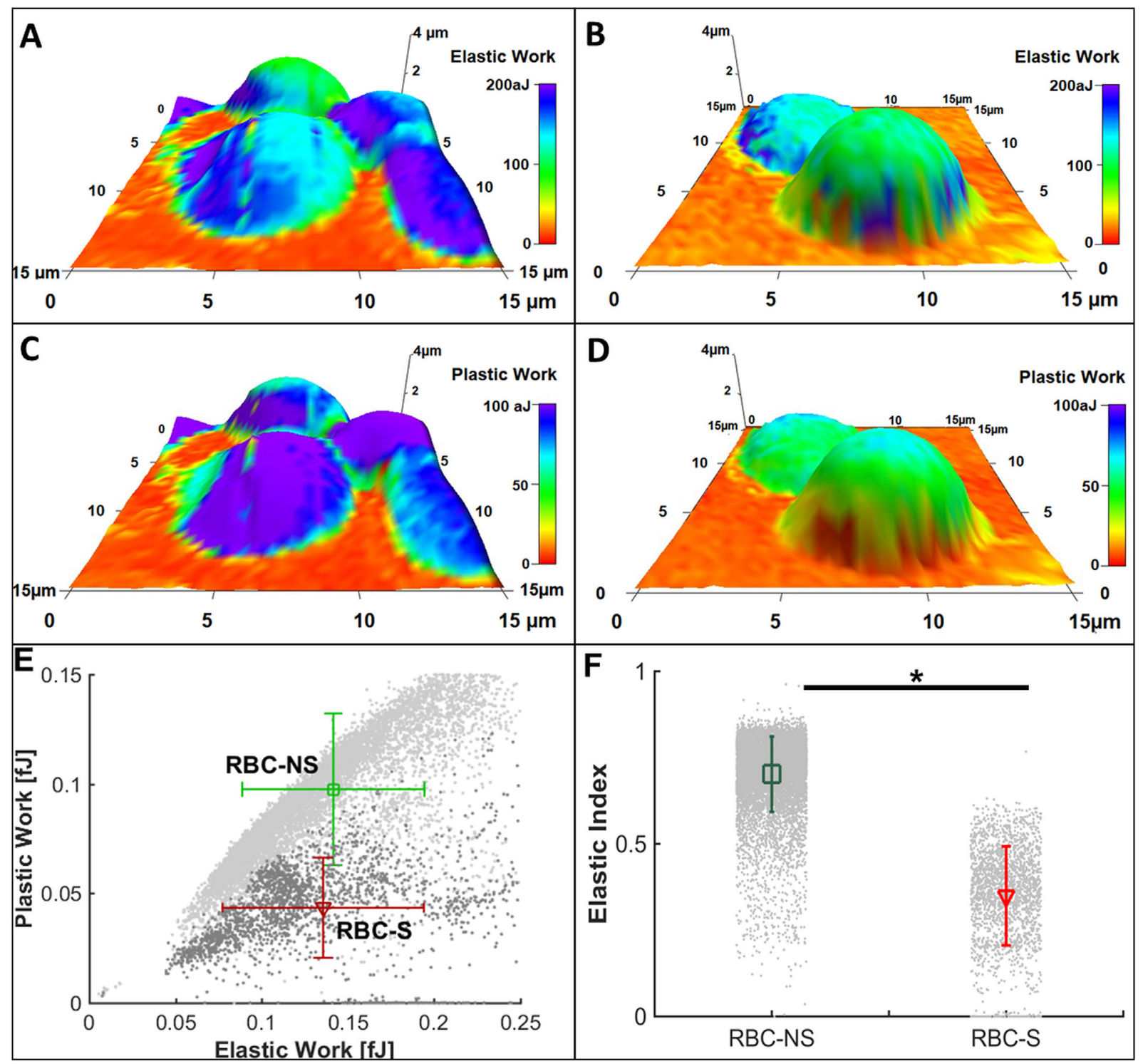

Fig. 2. Investigation on the rheological status of RBCs by atomic force microscopy.

Representative three-dimensional reconstructed topographies of RBC-NS (A and C) and RBC-S (B and D) pseudo-coloured with Elastic Work (A and B) and Plastic Work (C and D). Values of Plastic Work versus Elastic Work are shown as mean \pm standard deviation represented by error bars (E). The ratio of Plastic Work: Elastic Work denoted as Elastic Index is represented by average \pm standard deviation for RBC-NS $(\mathrm{n}=41)$ and RBC-S $(\mathrm{n}=32)$ cells $(\mathrm{F})$.

acid (PUFA)-containing lipid species, which are potential targets for lipid peroxidation. The results for the significantly affected species are summarized in Fig. 4E.

\subsection{Elevated 4-hydroxynonenal (4-HNE) formation confirms increased lipid peroxidation}

The major products of lipid peroxidation are oxygenated $\alpha$-, $\beta$-unsaturated aldehydes; one of the most studied being 4-HNE. To follow its level, we choose a widely accepted, specific detection method, the immunolabeling of the aldehyde-protein adduct with anti-4-HNE antibody. In agreement with the above-shown decrease of PUFA-containing structural lipids, we were able to detect significant increase in the level of 4-HNE adduct formation (8-10-fold) in the RBC-S group as compared to the RBC-NS (Fig. 5A and B).

\section{Discussion}

Sustained smoking-induced hypoxia, excessive generation of free radicals and drastic reduction of bioavailable NO initiates the development of pathologically altered vascular system [34,35]. An understanding about the pathophysiological mechanisms in the vascular diseases necessitates a detailed knowledge regarding the complications in the circulating RBCs. This project was based on the expectation that the state of the circulating RBCs in the developing fetus is reflective to the harmful substances that originated from an improper maternal lifestyle and remain unfiltered by the placental barrier.

Our results indicate that due to maternal smoking the physicochemical properties of fetal RBCs undergo significant alterations including elevated plasticity and altered lipid composition of the plasma membrane. We demonstrated that RBCs with smoking origin display significantly higher level of a toxic oxidant, $\mathrm{ONOO}^{-}$, generated by the spontaneous reaction of superoxide anion $\left(\mathrm{O}_{2} \cdot{ }^{-}\right)$and NO [36], which suggests an increased $\mathrm{O}_{2} \cdot{ }^{-}$generation and an alteration in the bioavailability and actions of NO. Compared to the control values, the $\mathrm{H}_{2} \mathrm{O}_{2}$ level was somewhat lower in the RBC-S population, which was paralleled with unaltered/decreased activities of scavenging enzymes like SOD and CAT. 


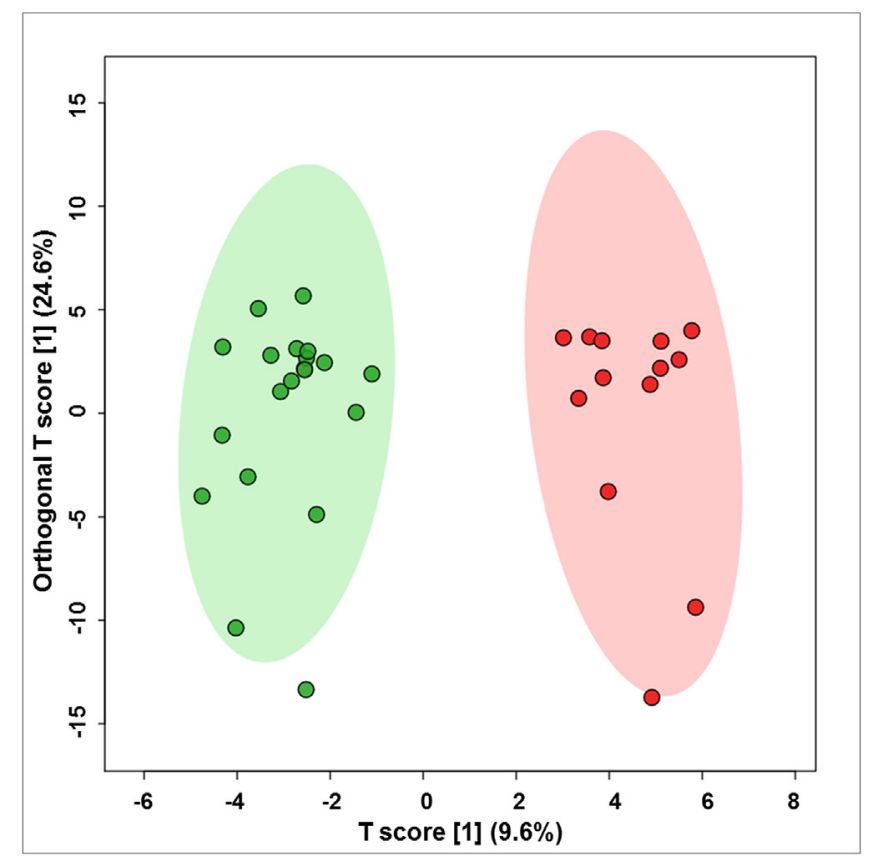

Fig. 3. Orthogonal partial least squares analysis scores plot of lipidomic data. Values are shown for the RBC-NS $(n=20$, green) and RBC-S $(n=13$, red) populations; goodness-of-prediction value was Q2 $=0.58(\mathrm{p}<0.001)$ and goodness-of-fit value was $\mathrm{R} 2=0.94(\mathrm{p}<0.001)$ based on $\mathrm{n}=1000$ permutation tests. Coloured circles display $95 \%$ confidence regions.

These results, together with the fact that $\mathrm{O}_{2} \cdot{ }^{-}$is a common partner for the formation of both ONOO- and $\mathrm{H}_{2} \mathrm{O}_{2}$, imply that the production of ONOO- is limited to the available NO instead of the $\mathrm{O}_{2} \cdot{ }^{-}$level. If so, this suggests the presence of an excess $\mathrm{O}_{2} \cdot{ }^{-}$content, which is alternatively able to reduce quinones and transition metals, thus inducing impairment of metal-containing enzymes. Furthermore, free $\mathrm{O}_{2} \cdot{ }^{-}$ contribute to hydroperoxyl radical formation, which by subtracting hydrogen atoms from PUFA and lipid hydroperoxides, similarly to $\mathrm{ONOO}^{-}$, initiates lipid oxidation [37]. Indeed, a significant increase in the formation of 4-HNE, a known product of lipid peroxidation was detected in RBC-S population. The increased frequency of lipid peroxidation indirectly signifies a loss of membrane integrity and function as well as an impairment in the rheological parameters.

The extensive deformation and relaxation properties of RBCs are determined by the membrane composition and properties of the membrane-cytoskeleton complex [38]. Multivariate statistics on our lipidomic dataset revealed that long-term maternal smoking leaves characteristic fingerprint on the lipidome of the RBC-S population compared to the RBC-NS. Furthermore, we could identify several interpretable differences. The elevated Chol/PL ratio in the RBC-S group points to a decrease in membrane fluidity; indeed, it was documented for adult RBCs that the increase in Chol/PL paralleled the decrease in membrane fluidity $[39,40]$. In addition, the decrease in the average degree of unsaturation of the RBC-S lipids also confirms that the RBC-S population possesses a more rigid membrane that can enhance the resistance to the hemodynamic flow. Under normal physiological conditions, Chol is equally distributed between the two membrane leaflets, whereas the 4 major PLs - the outer monolayer PC and SM and the inner monolayer PE and PS - are asymmetrically disposed [40]. We showed that the membrane lipid asymmetry differs between the RBC-NS and RBC-S populations. This derived mainly from the lowering of PS level. It was reported that the change in membrane lipid asymmetry itself may adversely influence the association of membrane proteins [41]. On the other hand, the reduction in PS may refer to the weakening of the interaction between the cytoskeletal network and the bilayer due to the known specific interaction of PS with spectrin proteins, thus causing a less fluid or flexible RBC-S membrane [42]. Finally, the significant decreases in the levels of several oxidation-prone, i.e., PUFA-containing lipid species in the RBCs with smoking origin confirm the elevated oxidative damage caused by ROS generation. As mentioned above, this loss on the target side paralleled the significant increase on the product side, i.e., the level of 4-HNE elevated as a result of lipid peroxidation in the RBC-NS group.

The nanomechanical property of deformability is highly determined by the elastic and plastic activities of the RBC membrane-cytoskeleton complex $[43,44]$. There are applied biophysical techniques to measure the RBCs' "membrane stiffness" like rheoscopy, ektacytometry or optical trapping. One of the most direct and robust techniques to investigate RBCs' rheological pattern close to native conditions is atomic force microscopy-based force spectroscopy $[44,45]$. In the RBC-S samples we registered increase in the membrane stiffness; similar findings were reported by Lekka and co-workers in adults under several pathological conditions like coronary disease, diabetes mellitus, and hypertension [46]. Additionally, we were able to characterize the viscoelastic response of RBCs to the mechanical stimulus, where we found a remarkable decrease in the recovering ability of RBCs with smoking origin after indentation. It is conceivable that the long-term exposure to the cytotoxic effectors derived from cigarette smoke markedly influenced the hemorheological properties of RBCs by architectural modifications which led to loss in the ability to regain their initial shape. As suggested by Pretorius and Masilamani, the increase of plastic work closely associated with cytoskeletal membrane stiffness and redox homeostasis imbalance [13,47].

The measured parameters in this work are well indicative to the vulnerability of the fetal circulatory system as a consequence of maternal smoking. Although the directly exposed RBCs derived from smoking mothers possess a functional and mature antioxidant system that still has the capacity to increase $\mathrm{O}_{2} \cdot{ }^{-}$scavenging activity under stress conditions [48], the endogenous antioxidant system in the fetal RBCs is barely functional; the delicate oxidant/antioxidant balance is disrupted. Lipid peroxidation, as measured by 4-HNE level, is almost doubled in the fetal red cells with smoking origin compared to the maternal population [48]. This prominently indicates a higher rate of macromolecular damage in the fetal system, via indirect exposure to tobacco smoke, which gives rise to all other consequences for the rheological features.

\section{Conclusion}

In this work we demonstrated that fetal RBCs are highly affected by maternal smoking; they become a source of ROS and lose their characteristic membrane and rheological features. The importance of our work is underlined by recent findings about a close relationship between the vascular endothelium and the circulating RBCs, which was demonstrated in patients with type 2 diabetes mellitus [11,12]. An excess level of toxic oxidants not only compromises the RBC function and viability, but the liberated reactive oxygen/nitrogen species from the considerable mass of $\mathrm{RBC}$ have a potential to influence other components of the circulation as well. Therefore, the induced lipid oxidation, the altered membrane lipid composition, membrane stiffness and loss in plastic activities could serve as an early prognostic markers for not only the RBC-linked anomalies, but also the state of the neonatal vascular environment. Furthermore, assuming that under stress conditions circulating RBCs are able to sense and respond to endothelial demands to improve the blood flow, it is most likely that in the RBC-S population the compensatory/rescue mechanism remained unavailable due to altered membrane integrity and rheological properties.

Supplementary data to this article can be found online at https:// doi.org/10.1016/j.bbalip.2020.158615. 


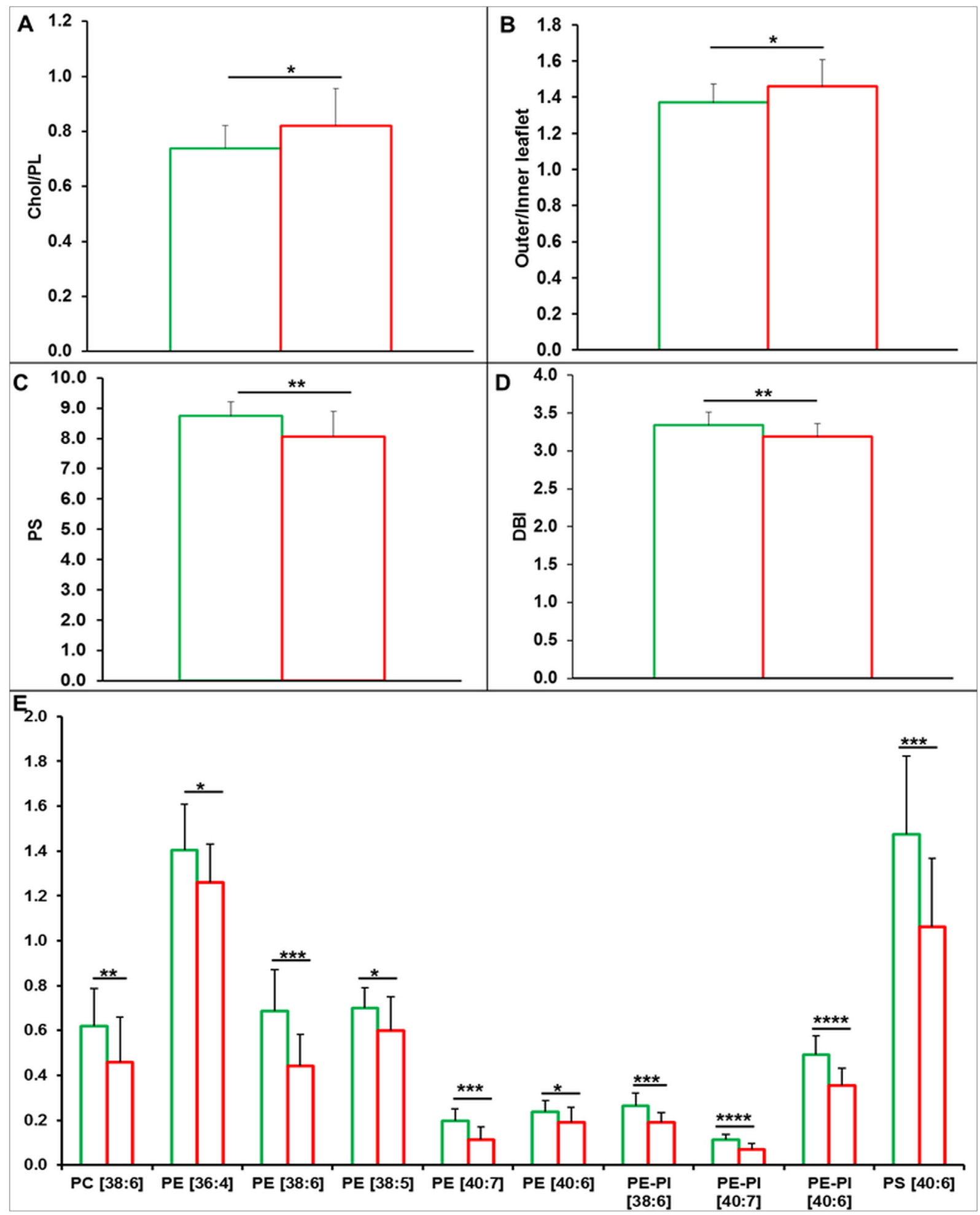

Fig. 4. Lipidomic differences between plasma membranes from the RBC-NS and RBC-S samples.

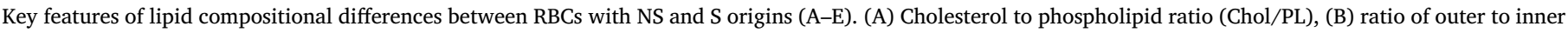

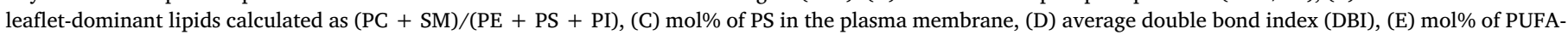

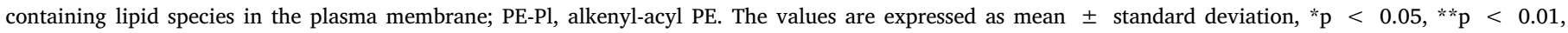
$* * * \mathrm{p}<0.001, * * * * \mathrm{p}<0.0001$.

\section{Funding}

This work was supported by the European Union and the Hungarian Government in the framework of the GINOP-2.3.2-15-2016-00040 project.

\section{Transparency document}

The Transparency document associated this article can be found, in online version. 

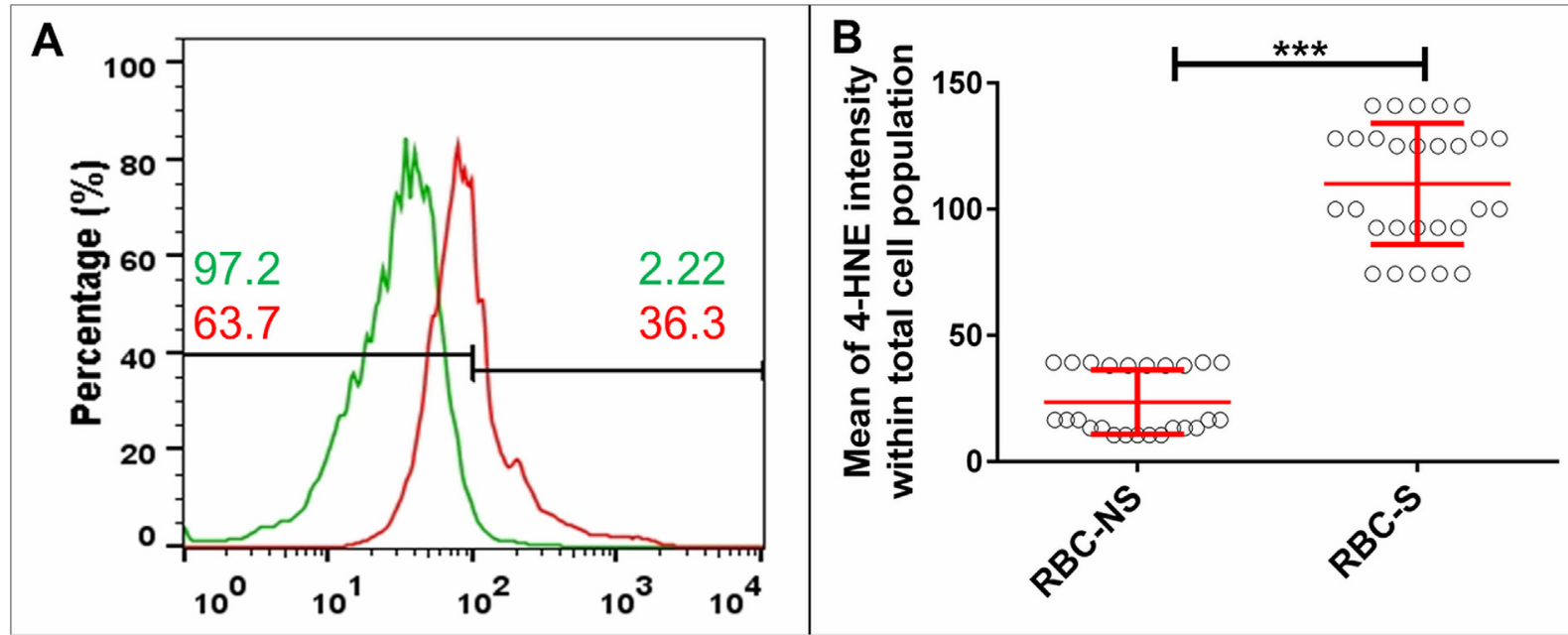

Fig. 5. Comparison of the 4-HNE level by FACS analysis between the RBC-NS and RBC-S populations.

Representative histogram (A) of the intensity level of 4-HNE from FACS analysis for the RBC-NS (green) and RBC-S (red) populations. The values below and above $10^{2}$ indicate the percentage distribution of the 4-HNE-positive cells. The graph (B) shows the 4-HNE level in the RBC NS ( $\left.\mathrm{n}=25\right)$ and RBC-S (n $\left.=28\right)$ populations. The results were accepted to be significant by statistical analyses of one-way ANOVA using the Newman-Keuls multiple comparison test at $* * * \mathrm{p}<0.001$.

\section{Declaration of competing interest}

No conflict of interest was reported by the authors.

\section{References}

1] G.P. Pfeifer, M.F. Denissenko, M. Olivier, N. Tretyakova, S.S. Hecht, P. Hainaut, Tobacco smoke carcinogens, DNA damage and p53 mutations in smoking-associated cancers, Oncogene. 21 (48) (2002 Oct 21) 7435-7451, https://doi.org/10. 1038/sj.onc.1205803.

2] B.C. Feltes, J. de F. Poloni, D.L. Notari, D. Bonatto, Toxicological effects of the different substances in tobacco smoke on human embryonic development by a systems chemo-biology approach, PLoS One 8 (4) (2013 Apr 29) e61743, , https:// doi.org/10.1371/journal.pone.0061743.

[3] D.F. Church, W.A. Pryor, Free-radical chemistry of cigarette smoke and its toxicological implications, Environ. Health Perspect. 64 (1985 Dec) 111-126, https:// doi.org/10.1289/ehp.8564111.

4] A. Hackshaw, C. Rodeck, S. Boniface, Maternal smoking in pregnancy and birth defects: a systematic review based on 173687 malformed cases and 11.7 million controls, Hum. Reprod. Update. 17 (5) (2011 Sep-Oct) 589-604, https://doi.org/ 10.1093/humupd/dmr022.

[5] I. Berlin, C. Oncken, Maternal smoking during pregnancy and negative health outcomes in the offspring, Nicotine Tob. Res. 20 (6) (2018 May 3) 663-664, https://doi.org/10.1093/ntr/nty035.

[6] J.A. Courtney, J.F. Cnota, H.N. Jones, The role of abnormal placentation in congenital heart disease; cause, correlate, or consequence? Front. Physiol. 9 (2018 Aug 7) 1045, https://doi.org/10.3389/fphys.2018.01045.

[7] G. Acharya, S.E. Sonesson, K. Flo, J. Räsänen, A. Odibo, Hemodynamic aspects of normal human feto-placental (umbilical) circulation, Acta Obstet. Gynecol. Scand. 95 (6) (2016 Jun) 672-682, https://doi.org/10.1111/aogs.12919.

[8] K.H. Al-gubory, P.A. Fowler, C. Garrel, The roles of cellular reactive oxygen species, oxidative stress and antioxidants in pregnancy outcomes, Int. J. Biochem. Cell Biol. 42 (10) (2010 Oct) 1634-1650, https://doi.org/10.1016/j.biocel.2010.06.001.

[9] P. Rodríguez-Rodríguez, D. Ramiro-Cortijo, C.G. Reyes-Hernández, A.L. López de Pablo, M. Carmen González, S.M. Arribas, Implication of oxidative stress in fetal programming of cardiovascular disease, Front. Physiol. 9 (2018 May 23) 602, https://doi.org/10.3389/fphys.2018.00602.

[10] M.M. Cortese-krott, M. Kelm, Endothelial nitric oxide synthase in red blood cells: key to a new erythrocrine function ? Redox Biol. 2 (2014 Jan 9) 251-258, https:// doi.org/10.1016/j.redox.2013.12.027.

[11] J. Pernow, A. Mahdi, J. Yang, Z. Zhou, Red Blood Cell Dysfunction: A New Player in Cardiovascular Disease, 115 (2019 June 10), pp. 1596-1605, https://doi.org/10. 1093/cvr/cvz156.

[12] Z. Zhou, A. Mahdi, Y. Tratsiakovich, S. Zahorán, O. Kövamees, F. Nordin, A.E. Uribe Gonzalez, M. Alvarsson, C.G. Östenson, D.C. Andersson, U. Hedin, E. Hermesz, J.O. Lundberg, J. Yang, J. Pernow, Erythrocytes from patients with type 2 diabetes induce endothelial dysfunction via Arginase I, J. Am. Coll. Cardiol. 72 (7) (2018 Aug 14) 769-780, https://doi.org/10.1016/j.jacc.2018.05.052.

[13] E. Pretorius, J.N. Du Plooy, P. Soma, I. Keyser, A.V. Buys, Smoking and fluidity of erythrocyte membranes: a high resolution scanning electron and atomic force microscopy investigation, Nitric Oxide 35 (2013 Nov 30) 42-46, https://doi.org/10 1016/j.niox.2013.08.003.

[14] J.A. Ambrose, R.S. Barua, The pathophysiology of cigarette smoking and cardiovascular disease: an update, J. Am. Coll. Cardiol. 43 (10) (2004 May 19)
1731-1737, https://doi.org/10.1016/j.jacc.2003.12.047

[15] M. Girasole, S. Dinarelli, G. Boumis, Structure and function in native and pathological erythrocytes: a quantitative view from the nanoscale, Micron. 43 (12) (2012 Dec) 1273-1286, https://doi.org/10.1016/j.micron.2012.03.019.

[16] E. Villegas, S.E. Gilliland, Hydrogen peroxide production by lactobacillus delbrueckii subsp. Lactis I at $5^{\circ} \mathrm{C}$, J. Food Sci. 63 (6) (1998 November) 1070-1074, https://doi.org/10.1111/j.1365-2621.1998.tb15857.x.

[17] R.E. Huie, S. Padmaja, The reaction of no with superoxide, Free Radic. Res. 18 (4) (1993 Jun 04) 195-199, https://doi.org/10.3109/10715769309145868.

[18] O.H. Lowry, N.J. Rosebrough, A.L. Farr, R.J. Randall, Protein measurement with the Folin phenol reagent, J. Biol. Chem. 193 (1) (1951 Nov) 265-275, https://doi.org/ 10.1016/0304-3894(92)87011-4.

[19] H.P. Misra, I. Fridovich, The role of superoxide anion in the autoxidation of epinephrine and a simple assay for superoxide dismutase, J. Biol. Chem. 247 (10) (1972 May 25) 3170-3175.

[20] R.F. Beers Jr., I.W. Sizer, Catalase assay with special reference to manometric methods, Science. 117 (3052) (1953 Jun 26) 710-712, https://doi.org/10.1126/ science.117.3052.710-a.

[21] M. Péter, A. Glatz, P. Gudmann, I. Gombos, Z. Török, I. Horváth, L. Vígh, G. Balogh, Metabolic crosstalk between membrane and storage lipids facilitates heat stress management in Schizosaccharomyces pombe, PLoS One 12 (3) (2017 Mar 10) 1-22, https://doi.org/10.1371/journal.pone.0173739.

[22] B. Peksel, I. Gombos, M. Péter, L. Vigh Jr., Á. Tiszlavicz, M. Brameshuber, G. Balogh, G.J. Schütz, I. Horváth, L. Vigh, Z. Török, Mild heat induces a distinct "eustress" response in Chinese Hamster Ovary cells but does not induce heat shock protein synthesis, Sci. Rep. 7 (15643) (2017 November 15) 15821-15828, https:// doi.org/10.1038/s41598-017-15821-8.

[23] G. Liebisch, M. Binder, R. Schifferer, T. Langmann, B. Schulz, G. Schmitz, High throughput quantification of cholesterol and cholesteryl ester by electrospray ionization tandem mass spectrometry (ESI-MS/MS), Biochim. Biophys. Acta 1761 (1) (2006 January) 121-128, https://doi.org/10.1016/j.bbalip.2005.12.007.

[24] R. Herzog, D. Schwudke, K. Schuhmann, J.L. Sampaio, S.R. Bornstein, M. Schroeder, A. Shevchenko, A novel informatics concept for high-throughput shotgun lipidomics based on the molecular fragmentation query language, Genome Biol. 12 (R8) (2011 January 19), https://doi.org/10.1186/gb-2011-12-1-r8.

[25] G. Liebisch, J.A. Vizcaíno, H. Köfeler, M. Trötzmüller, W.J. Griffiths, G. Schmitz, F. Spener, M.J. Wakelam, Shorthand notation for lipid structures derived from mass spectrometry, J. Lipid Res. 54 (6) (2013 Jun) 1523-1530, https://doi.org/10.1194/ jlr.M033506.

[26] R. Zeller, et al., Fixation, embedding, and sectioning of tissues, embryos, and single cells, Curr Protoc Pharmacol. (2001 May) 3D, https://doi.org/10.1002/ 0471141755.pha03ds07 Appendix3.

[27] J.E. Sader, J.A. Sanelli, B.D. Adamson, J.P. Monty, X. Wei, S.A. Crawford, J.R. Friend, I. Marusic, P. Mulvaney, E.J. Bieske, Spring constant calibration of atomic force microscope cantilevers of arbitrary shape, Rev Sci Instrum. 83 (10) (2012 October) 103705, https://doi.org/10.1063/1.4757398.

[28] J.L. Hutter, J. Bechhoefer, Calibration of atomic-force microscope tips, Rev. Sci. Instrum. 64 (7) (1998 September 09) 1868-1873, https://doi.org/10.1063/1. 1143970.

[29] B. Varga, C. Fazakas, J. Molnár, I. Wilhelm, R.A. Domokos, I.A. Krizbai, Z. Szegletes, G. Váró, A.G. Végh, Direct mapping of melanoma cell - endothelial cell interactions, J. Mol. Recognit. 30 (2016 December 23), https://doi.org/10.1002/jmr.2603.

[30] B. Varga, R.A. Domokos, C. Fazakas, I. Wilhelm, I.A. Krizbai, Z. Szegletes, C. Gergely, G. Váró, A.G. Végh, De-adhesion dynamics of melanoma cells from brain endothelial layer, Biochim. Biophys. Acta - Gen. Subj. 1862 (3) (2018 March) 
745-751, https://doi.org/10.1016/j.bbagen.2017.10.013.

[31] D.F. Williamson, R.A. Parker, J.S. Kendrick, The box plot: a simple visual method to interpret data, Ann. Intern. Med. Ann Intern Med. 110 (11) (1989 June 1) 916-921, https://doi.org/10.7326/0003-4819-110-11-916.

[32] J. Xia, D.S. Wishart, Using metaboanalyst 3.0 for comprehensive metabolomics data analysis, Curr. Protoc. Bioinformatics 55 (2016 Sep 7) 14.10.1-14.10.91, https:// doi.org/10.1002/cpbi.11.

[33] N. Yeow, R.F. Tabor, G. Garnier, Atomic force microscopy: from red blood cells to immunohaematology, Adv. Colloid Interf. Sci. 249 (2017 Nov) 149-162, https:// doi.org/10.1016/j.cis.2017.05.011.

[34] M.S. Alsalhi, S. Devanesan, K.E. Alzahrani, M. Alshebly, F. Al-Qahtani, K. Farhat, V. Masilamani, Impact of diabetes mellitus on human erythrocytes: atomic force microscopy and spectral investigations, Int. J. Environ. Res. Public Health 15 (11) (2018 Nov) 2368, https://doi.org/10.3390/ijerph15112368.

[35] J.Y. Chen, Z.X. Ye, X.F. Wang, J. Chang, M.W. Yang, H.H. Zhong, F.F. Hong, S.L. Yang, Nitric oxide bioavailability dysfunction involves in atherosclerosis, Biomed. Pharmacother. 97 (2018 Jan) 423-428, https://doi.org/10.1016/j.biopha. 2017.10.122.

[36] R. Radi, Oxygen radicals, nitric oxide, and peroxynitrite: redox pathways in molecular medicine, Proc. Natl. Acad. Sci. U. S. A. 115 (23) (2018 Jun 5) 5839-5848, https://doi.org/10.1073/pnas.1804932115.

[37] N.M. Burton, L.J. Bruce, Modelling the structure of the red cell membrane, Biochem. Cell Biol. 89 (2) (2011 March 16) 200-215, https://doi.org/10.1139/ O10-154.

[38] J.S. Owen, K.R. Bruckdorfer, R.C. Day, N. McIntyre, Decreased erythrocyte membrane fluidity and altered lipid composition in human liver disease, J. Lipid Res. 23 (1) (1982 Jan) 124-132.

[39] V. Kuhn, L. Diederich, T.C.S. Keller, C.M. Kramer, W. Lückstädt, C. Panknin, T. Suvorava, B.E. Isakson, M. Kelm, M.M. Cortese-Krott, Red blood cell function and dysfunction: redox regulation, nitric oxide metabolism, anemia, Antioxid. Redox Signal. 26 (13) (2017 May 1) 718-742, https://doi.org/10.1089/ars.2016.6954.

[40] N. Mohandas, P.G. Gallagher, Red cell membrane: past, present, and future, Blood.
112 (10) (2008 Nov 15) 3939-3948, https://doi.org/10.1182/blood-2008-07161166.

[41] Y. Chico, M. Lafita, P. Ramírez-Duque, F. Merino, B. Ochoa, Alterations in erythrocyte membrane lipid and fatty acid composition in Chediak-Higashi syndrome, Biochim. Biophys. Acta 1502 (3) (2000 Nov 15) 380-390, https://doi.org/10.1016/ S0925-4439(00)00062-4.

[42] S. Manno, Y. Takakuwa, N. Mohandas, Identification of a functional role for lipid asymmetry in biological membranes: phosphatidylserine-skeletal protein interactions modulate membrane stability, Proc. Natl. Acad. Sci. U. S. A. 99 (4) (2002 Feb 19) 1943-1948, https://doi.org/10.1073/pnas.042688399.

[43] J. Kim, H.Y. Lee, S. Shin, Advances in the measurement of red blood cell deformability: a brief review, J. Cell. Biotechnol. 1 (1) (2015) 63-79, https://doi.org/10. 3233/JCB-15007.

[44] F.S. Ruggeri, C. Marcott, S. Dinarelli, G. Longo, M. Girasole, G. Dietler, T.P.J. Knowles, Identification of oxidative stress in red blood cells with nanoscale chemical resolution by infrared nanospectroscopy, Int. J. Mol. Sci. 19 (9) (2018 Aug 30), https://doi.org/10.3390/ijms19092582.

[45] G. Ciasca, M. Papi, S. Di Claudio, M. Chiarpotto, V. Palmieri, G. Maulucci, G. Nocca, C. Rossi, M. De Spirito, Mapping viscoelastic properties of healthy and pathological red blood cells at the nanoscale level, Nanoscale. 7 (40) (2015 Oct 28) 17030-17037, https://doi.org/10.1039/c5nr03145a.

[46] M. Lekka, M. Fornal, G. Pyka-Fościak, K. Lebed, B. Wizner, T. Grodzicki, J. Styczeń, Erythrocyte stiffness probed using atomic force microscope, Biorheology. 42 (4) (2005) 307-317.

[47] V. Masilamani, K. Alzahrani, S. Devanesan, H. Alqahtani, M.S. AlSalhi, Smoking induced hemolysis: spectral and microscopic investigations, Sci. Rep. 19 (6) (2016 February) 21095, https://doi.org/10.1038/srep21095.

[48] P. Chakraborty, K.N. Dugmonits, A.G. Végh, R. Hollandi, P. Horváth, J. Maléth, P. Hegyi, G. Németh, E. Hermesz, Failure in the compensatory mechanism in red blood cells due to sustained smoking during pregnancy, Chem. Biol. Interact. 313 (2019 Nov 1) 108821, https://doi.org/10.1016/j.cbi.2019.108821. 